\title{
Das Besondere der Hausarztmedizin
}

\section{Corinne Bertschi}

Ärztin in Weiterbildung zur Hausärztin
Korrespondenz:

Med. pract. Corinne Bertschi Seestrasse 79

CH-3800 Unterseen

corinne.bertschi@jhas.ch
Ich sitze vor dem Computer, es ist vier Uhr in der Früh und der grobe Ansturm auf die Notfallstation im Spital hoffentlich vorbei. Endlich habe ich Zeit, die sieben stationär aufgenommenen Patienten dieser Nachtschicht nicht nur in meinem Kopf, sondern auch im Computersystem zu ordnen, ihre Diagnoselisten anzupassen, vergessengegangene Verordnungen nachzuholen und etliche Anmeldungen auszufüllen. Es ist die vorletzte von sieben aufeinanderfolgenden Nachtschichten und so etliche meiner Hirnzellen melden einen halbleeren Batteriestand. In der Stille der Nacht, neben dem Brummen des Computers, driften meine Gedanken plötzlich ab ...

Ich erinnere mich an meine viermonatige Praxisassistenz, die ich nach dem Staatsexamen vor dem Eintauchen in die Spitalmedizin in einer Einzelpraxis absolviert habe. Eigentlich war es ein Lückenfüller, um etwas Geld zu verdienen und mein Wissen nach dem Staatsexamen frisch zu halten. Rückblickend war dies jedoch eine der wegweisenden Erfahrungen in meiner bisher kurzen medizinischen Karriere.

\section{Kleine Taten und ihre Wirkung}

Ich erinnere mich zum Beispiel an einen Patienten mittleren Alters, der zweiwöchentlich pünktlich um

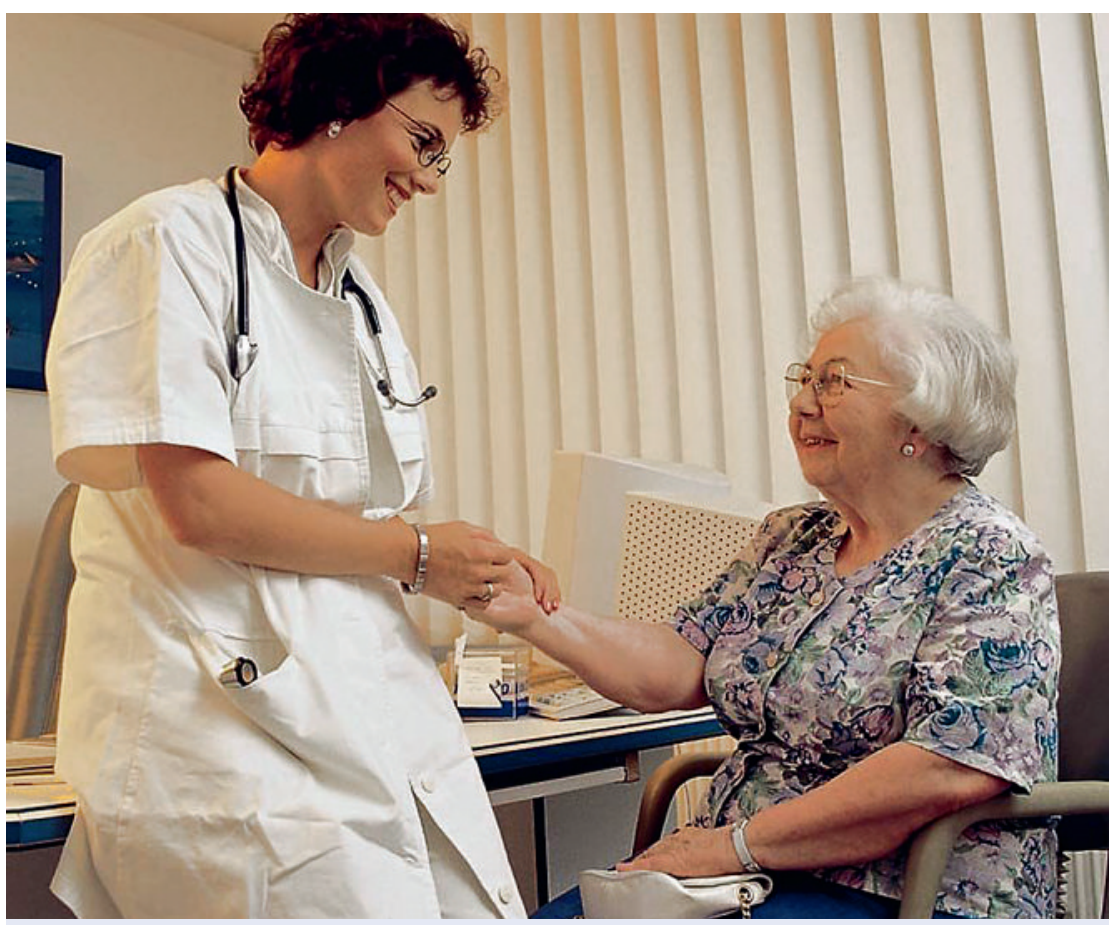

Genügend Zeit haben für «das andere» neben dem rein Medizinischen - ein wichtiges Ziel für junge Hausärzte.
13.25 Uhr im Wartezimmer sass und mich anstrahlte, wenn ich von der Mittagspause zurückkam. Während der jeweiligen Injektion des intramuskulären Depotpräparates erzählte er mir so einiges aus seinem Alltag im Heim. Zum Beispiel, dass er die Fleischpastetli, die er sich als Geburtstagsmenu wünschen konnte, besonders genossen hätte. Ein anderes Mal, dass er zur Silvesterparty eine Flasche Rimuss erhalten hätte und so trotz Einnahme vieler Medikamente «richtig» hätte anstossen dürfen. Es waren Konsultationen, die kaum zehn Minuten dauerten, und doch hatte da so vieles Platz. An meinem letzten Arbeitstag drückte er mir

\section{«Wann kommen Sie wieder zurück?»}

fünf Franken in die Hand und zwinkerte mir mit strahlenden Augen zu: «Für einen Kafi, Sie waren sehr nett und von Ihren Injektionen habe ich nie etwas gespürt! Wann kommen Sie wieder zurück?» Solche Bestätigungen sind eine Wohltat, denn Patienten zufrieden zu sehen, ist und bleibt eines meiner höchsten Ziele. Medizinisch betrachtet waren diese zweiwöchentlichen Depotinjektionen zwar kleine Taten, doch von grösserer Bedeutung. Nach den insgesamt acht Konsultationen, die ich bei diesem Patienten durchgeführt hatte, war bereits eine Bindung herangewachsen. Wie solche Bindungen nach 20 Jahren Praxistätigkeit aussehen mögen, wage ich kaum zu erahnen.

\section{Aus dem Alltäglichen das Besondere entnehmen}

Oder da war eine Dame mittleren Alters, die während vier Wochen regelmässig zur Warzenbehandlung kam. Während ich an ihren Füssen und Händen manipulierte, ergaben sich nette Gespräche. Zum Beispiel über ihre Reise nach Lissabon, wobei ich hilfreiche Tipps für meine eigene Reise erhielt. Kürzlich, einige Monate nach Abschluss meiner Praxisassistenz, traf ich die Patientin per Zufall in der Stadt und sie erkundigte sich nach meinem Wohlbefinden und wie denn meine Reise nach Lissabon gewesen sei. Auch hier: Es hat viel mehr als nur die Warzenbehandlung stattgefunden.

\section{Hausarztluft zu schnuppern, kann sämtliche Pläne durcheinanderbringen}

Diese zwei Geschichten sehe ich sinnbildlich für die Hausarztmedizin. Sie ist viel mehr als Spritzen setzen 
und Warzen behandeln. Auch im Spital sollte genug Platz für «das andere» neben dem rein Medizinischen sein, doch leider fehlt dazu oft die Zeit. Während den Visiten stehen Vitalparameter, Medikamenteneinstellungen, Laborwerte sowie die Dynamik der Symptome im Vordergrund. Danach geht es an das Ordnen
Eine fundierte, breite Weiterbildung im Spital ist unerlässlich für uns zukünftige Hausärztinnen und -ärzte, und ich möchte diese Zeit auf keinen Fall missen. Danach in der Hausarztpraxis wird nicht alles einfacher und besser sein, aber «anders». Und auf dieses «andere» freue ich mich sehr. Ich bin dankbar,

\section{Rückblickend war die Praxisassistenz eine der wegweisenden Erfahrun- gen in meiner bisher kurzen medizinischen Karriere}

von Diagnosen, Anfordern von ausstehenden Resultaten und Schreiben von Austrittsberichten.

Der Computer neben mir brummt immer noch, in der Diagnosenliste bin ich bei Punkt 7 von 14 angekommen - und bereits klingelt das Diensttelefon wieder. Herr K. geistere verwirrt umher, habe sich das Venflon gezogen, die Pflegefachfrau traue sich wegen fliegenden Gegenständen nicht mehr ins Zimmer ... dass ich dies durch meine Praxisassistenz schon so früh in meiner Weiterbildungszeit erfahren durfte.

An dieser Stelle möchte ich alle Assistenzärztinnen und -ärzte dazu ermuntern, auch einmal - und sei es nur für einige Wochen - Hausarztluft zu schnuppern. Aber Achtung, es könnte sämtliche bisherigen Pläne durcheinanderbringen!

\section{Lust auf mehr Hausarztmedizin? \\ JHaS-Kongress am 2. April 2011}

Hausarzt werden?

Wie oft hast du dir diese Frage gestellt angesichts des heutigen Hausarztbilds, der unzureichend «praxisorientierten» Weiterbildung, des hausarztwidrigen Windes der Gesundheitspolitik, angesichts des Einzelkämpfertums in allen Bereichen? Nichtsdestotrotz...

\section{Hausarzt werden!}

$\mathrm{Zu}$ diesem Schluss sind auch motivierte junge Hausärzte, Assistenzärzte und Studenten gekommen, allerdings mit einer Bedingung: Allein, nein! So war die Idee des verbindenden Vereins der Jungen Hausärztinnen und -ärzte Schweiz (JHaS) geboren und der Impuls geschaffen, einen eigens für junge und zukünftige Hausärzte zugeschnittenen Kongress zu organisieren.

Am 1. JHaS-Kongress am 2. April 2011 treffen wir uns: Studierende, Assistenzärzte, junge Hausärzte und Interessierte der Hausarztmedizin. Zusammen wollen wir Neues erfahren, Wissen vermitteln, Fertigkeiten trainieren und unsere gemeinsame Motivation pflegen: Hausarzt werden? Hausarzt werden!

Datum: 2. April 2011

Ort: Altes Spital Solothurn

Anmeldung und Informationen: www.congress-info.ch/jhas 2011 oder www.jhas.ch 